\title{
EVALUACIÓN DE LA CALIDAD DEL AGUA DEL RÍO PITA (ECUADOR), IMPLICACIÓN PARA LA CONSERVACIÓN DE LA VIDA ACUÁTICA Y SILVESTRE
}

\section{WATER QUALITY ASSESSMENT OF PITA RIVER (ECUADOR), IMPLICATIONS FOR AQUATIC LIFE PRESERVATION}

\author{
Karina Simbaña-Farinango ${ }^{1 *}$, David Romero-Estévez ${ }^{1}$, \\ Gabriela Yánez-Jácome' ${ }^{1}$ David Benavides² \& Hugo Navarrete ${ }^{1}$
}

Recibido: 30 de abril 2019 / Aceptado: 19 de junio 2019

DOI: 10.26807/ia.v7i2.104

Palabras clave: contaminación, fenoles, hierro, índice de calidad del agua, metales pesados, TULSMA.

Keywords: contamination, environmental quality index, heavy metals, iron, phenols, TULSMA.

\section{RESUMEN}

El presente estudio corresponde a la evaluación de la calidad del agua del río Pita y sus afluentes ubicados dentro y fuera del Parque Nacional Cotopaxi. Se fijaron siete estaciones de monitoreo, en las cuales se recolectaron muestras

1CESAQ-PUCE, Quito, Ecuador (dfromero@puce.edu.ec; hnavarrete@puce.edu.ec; gsyanez@puce.edu.ec;

*correspondencia: kjsimbanaf@puce.edu.ec).

2 UISEK, Quito, Ecuador (geogravid@yahoo.com). 
aguas arriba y abajo de cada afluente, en abril de 2018 y abril de 2019. Se midieron parámetros físicoquímicos in situ como: $\mathrm{pH}$, temperatura y porcentaje de saturación de oxígeno disuelto (OD); se analizaron los parámetros establecidos en la normativa ambiental ecuatoriana: Texto Unificado de la Legislación Secundaria del Ministerio del Ambiente, TULSMA, Anexo 1, Libro VI, tabla 2, aplicando métodos de análisis de acuerdo al Standard Methods for the Examination of Water and Wastewater (APHA). Los resultados indican que las muestras recolectadas superan el límite permisible para fenoles $\left(0,001 \mathrm{mg} \cdot \mathrm{dm}^{-3}\right)$ establecido en el TULSMA, a excepción del río Hualpaloma, mientras que para el caso de hierro, todas las muestras del cauce del río Pita y sus afluentes sobrepasan el límite permisible $\left(0,3 \mathrm{mg} \cdot \mathrm{dm}^{-3}\right)$. A partir de los resultados obtenidos, las muestras recolectadas del río Pita cumplen con los criterios de calidad de agua establecidos para la conservación de la vida acuática y silvestre, a pesar de atravesar zonas urbanas, donde diariamente existen descargas domésticas e industriales por tratarse de un canal abierto. Finalmente, de acuerdo al índice de calidad del agua aplicado (ICA-NSF), los afluentes ubicados dentro del parque Nacional Cotopaxi presentaron una "calidad buena", mientras que los afluentes ubicados fuera del Parque Nacional presentaron una "calidad media" relacionada con la cercanía a zonas urbanas.

\section{ABSTRACT}

The present study corresponds to the evaluation of the water quality of the Pita river and its affluents located inside and outside the Cotopaxi National Park. Seven monitoring stations were set up, in which samples were collected upstream and downstream of each tributary, on April 2018 and April 2019. Physical-chemical parameters were measured in situ such as: $\mathrm{pH}$, temperature and percentage of dissolved oxygen saturation (OD); the parameters established in the Ecuadorian environmental regulations were analyzed: Unified Text of the Secondary Legislation of the Ministry of the Environment, TULSMA, Annex 1, Book VI, table 2, applying methods of analysis according to the Standard Methods for the Examination of Water and Wastewater (APHA). The results indicate that the collected samples exceed the permissible limit for phenols $(0.001$ $\mathrm{mg} \cdot \mathrm{dm}-3$ ) established in the TULSMA, with the exception of the Hualpaloma 
river, while for the case of iron, all the samples of the Pita riverbed and its affluents exceed the permissible limit $(0.3 \mathrm{mg} \cdot \mathrm{dm}-3)$. From the results obtained, the samples collected from the Pita river meet the water quality criteria established for the conservation of aquatic and wild life, despite crossing urban areas, where there are daily domestic and industrial discharges because it is an open channel. Finally, according to the Quality Index applied (ICA-NSF), the affluents located within the Cotopaxi National Park presented a "good quality", while the affluents located outside the National Park presented an "average quality" related to the proximity to urban areas.

\section{INTRODUCCIÓN}

En el Ecuador existe una importante red hidrográfica originada principalmente en la cordillera de los Andes, y que desemboca en el río Amazonas y Océano Pacífico (Liñero et al., 2016). Actualmente, la mayoría de los ríos presentan grandes impactos antrópicos (Liñero et al., 2016), debido al crecimiento poblacional, la descarga de desechos, producción de agua potable, riego, actividades artesanales y ganaderas, cultivo intensivo de la tierra para satisfacer las exigencias de los consumidores y el desarrollo industrial (Jacobsen, 2003; Leandro et al., 2010). Los principales contaminantes de los cauces de agua son compuestos orgánicos volátiles, biodegradables, recalcitrantes, metales tóxicos, nutrientes vegetales, sólidos en suspensión, patógenos microbianos y parásitos, entre otros (Paul, 2017); estos contaminantes destruyen o modifican la fauna y la flora existente, superando en la mayoría de los casos la capacidad de auto-depuración propia de los sistemas hídricos (Menéndez, 2010).

El río Pita es una de las principales fuentes hídricas que abastece de agua potable al Distrito Metropolitano de Quito, este río forma parte de la cuenca alta del río Guayllabamba, la cual a su vez es parte de la cuenca del río Esmeraldas que desemboca en las costas del Pacífico ecuatoriano (FONAG, 2014). La mayor parte del caudal del río Pita nace en los páramos occidentales del volcán Sincho- 
lagua y una parte de los deshielos del volcán Cotopaxi (Proaño, 2007).

La topografía del río Pita está marcada por la presencia de los volcanes Cotopaxi y Sincholagua, y quebradas menores. Al final de su cauce, atraviesa el Valle de los Chillos hasta desembocar en el río San Pedro, a una altitud de 2.440 metros sobre el nivel del mar (msnm), en el sector sureste del cerro Ilaló, luego de recorrer aproximadamente $44 \mathrm{~km}$ en sentido sureste-noroeste (MAE, 2012). A medida que disminuye la altitud a lo largo del río Pita, este se encuentra cada vez más influenciado por actividades antropogénicas, como: la captación de agua para riego o potabilización, descargas directas de aguas servidas y desechos provenientes de cuencas de drenaje urbano e industrial, actividades agrícolas, florícolas y ganaderas, zonas destinadas al turismo, entre otras (Jacobsen, 2003; Campaña et al., 2017). Todas estas actividades pueden contribuir con la contaminación del río en sitios específicos debido a descargas líquidas o desechos sólidos presentes (Campaña et al., 2017).
El uso adecuado y cuidado del agua pueden conservar este recurso natural y su calidad innata (COA, 2017; Hernandez \& Lara, 2005); en base a esto el Ministerio del Ambiente del Ecuador (MAE) y las autoridades correspondientes han establecido normativas regulatorias para la prevención y control de la contaminación ambiental, establecidas en el Texto Unificado de la Legislación Secundaria del Ministerio del Ambiente, TULSMA, figurado en el Anexo 1, Libro VI, titulado "Norma de calidad Ambiental y de descarga de efluentes al recurso agua". Este documento presenta los límites permisibles de los parámetros físicoquímicos de control de la calidad del agua, los cuales han sido determinados en función de su uso (MAE, 2017).

Así mismo, el MAE conjuntamente con la Empresa Pública Metropolitana de Agua Potable y Saneamiento de Quito (EPMAPS) y el Fondo para la Protección del Agua (FONAG), mantienen monitoreos constantes del manejo y conservación de las cuencas que aportan a los sistemas de agua potable (EMAPS, 2016; FONAG, 2014). 
Actualmente existen diferentes protocolos para evaluar la calidad del agua de los ríos, entre estos, el monitoreo biológico que emplea macro invertebrados acuáticos (Liñero et al., 2016), y también métodos físicoquímicos (Blanco, 2009; Leandro et al., 2010; Ríos et al., 2001; Rivera et al., 2004). Sin embargo, existen metodologías más utilizadas a nivel mundial, dentro de estas se tiene a los "índices de calidad del agua". El índice de calidad del agua de la Fundación Nacional de Saneamiento (ICA-NSF) de los Estados Unidos (Castro et al., 2015), es un índice que no relaciona el posible uso que se le dará al agua, y los métodos utilizados para determinar las características físicoquímicas y microbiológicas. Este instrumento permite identificar el deterioro o mejora de la calidad del agua de forma cualitativa (Maritza et al., 2016). Para el cálculo de este índice se utilizan nueve parámetros como variables de respuesta, los cuales son: potencial de hidrógeno $(\mathrm{pH})$, temperatura, porcentaje de saturación de oxígeno di- suelto (OD), turbiedad, coliformes fecales (de forma cuantitativa), demanda bioquímica de oxígeno $\left(\mathrm{DBO}_{5}\right)$, fosfato, nitratos y sólidos totales (Fernandez et al., 2005; Quiroz et al., 2017).

El objetivo de esta investigación fue evaluar la calidad del agua del río Pita en los meses de abril de dos años consecutivos 2018 y 2019, mediante la determinación de la presencia y concentración de los diferentes parámetros físicoquímicos, de acuerdo a la normativa ecuatoriana vigente TULSMA, Anexo 1, Tabla 2: "Criterios de calidad admisibles para la preservación de la vida acuática y silvestre en aguas dulces, marinas y estuarios", considerando los criterios de calidad para agua dulce; con la finalidad de identificar los puntos de contaminación y evaluar el cumplimien- to de la normativa de calidad ambiental y además, calcular el índice de calidad de agua, ICA-NSF, para conocer el deterioro o conservación del mismo.

\section{MATERIALES Y MÉTODOS}

Se recolectaron un total de 48 muestras (12 en abril del 2018 y 36 en abril del 2019). Las muestras fueron recolectadas durante época lluviosa. 
Los sitios de toma de muestra fueron establecidos a lo largo del cauce del río Pita, de acuerdo a la afluencia de las principales vertientes y su accesibilidad. Para este estudio se consideraron 7 puntos predominantes de toma de muestra (Tabla 1), se recolectaron muestras de aguas arriba y abajo de cada afluente. Se dividió el cauce del río en dos secciones, la primera correspondiente al segmento dentro del Parque Nacional Cotopaxi, que en su inicio recibe el aporte del río Hualpaloma, quebrada Madero y La Merced, y la segunda correspondiente al segmento fuera del Parque Nacional, recibiendo el aporte de las quebradas Chorro y Cariacu y del río Guapal, y finalmente se recolectó una muestra antes de la confluencia del río San Pedro.

\section{Tabla 1. Coordenadas geográficas de los puntos de muestreo} a lo largo del cauce del río Pita

\begin{tabular}{|c|c|c|c|}
\hline Punto & $\begin{array}{l}\text { Río/quebrada } \\
\text { aportante al río Pita }\end{array}$ & Longitud & Latitud \\
\hline 1 & Río Hualpaloma & $78^{\circ} 22^{\prime} 51.67^{\prime \prime} \mathrm{O}$ & $0^{\circ} 37^{\prime} 20.14^{\prime \prime} \mathrm{s}$ \\
\hline 2 & Quebrada Madero & $78^{\circ} 23^{\prime} 25.85^{\prime \prime} \mathrm{O}$ & $0^{\circ} 36^{\prime} 41.64 " \mathrm{~s}$ \\
\hline 3 & Quebrada Merced & $78^{\circ} 25^{\prime} 40.22^{\prime \prime} \mathrm{O}$ & $0^{\circ} 34^{\prime} 3.24 " S$ \\
\hline 4 & Quebrada Chorro & $78^{\circ} 25^{\prime} 57.86^{\prime \prime} \mathrm{O}$ & $0^{\circ} 29^{\prime} 9.73^{\prime \prime S}$ \\
\hline 5 & Quebrada Cariacu & $78^{\circ} 23^{\prime} 46.50^{\prime \prime} \mathrm{O}$ & $0^{\circ} 23^{\prime} 23.73 " \mathrm{~s}$ \\
\hline 6 & Río Guapal & $78^{\circ} 23^{\prime} 46.50^{\prime \prime} \mathrm{O}$ & $0^{\circ} 23^{\prime} 20.11$ "s \\
\hline 7 & $\begin{array}{l}\text { Antes de la confluencia } \\
\text { del río San Pedro }\end{array}$ & $78^{\circ} 27^{\prime} 46.80^{\prime \prime} \mathrm{O}$ & $0^{\circ} 17^{\prime} 27.21 " \mathrm{~s}$ \\
\hline
\end{tabular}

Se analizaron parámetros in situ como: temperatura, $\mathrm{pH}$ y porcentaje de saturación de OD, utilizando un multiparámetros $(\mathrm{HACH}$, modelo HQ40d), para las condiciones ambientales temperatura y humedad relativa se utilizó un medidor ambiental (Control Company, modelo 1227U10). Las muestras fueron recolectadas $y$ preservadas según lo descrito en la norma NTE INEN 2169:2013 (INEN, 2013), para posteriormente ser transportadas al Centro de Estudios Aplicados en Química, CESAQ, y analizar los siguientes parámetros: aluminio $(\mathrm{Al})$, arsénico (As), bario (Ba), boro $(B)$, manganeso $(\mathrm{Mn})$, cianuro libre $\left(\mathrm{CN}^{-}\right)$, demanda bioquímica de oxí- 
geno durante cinco días $\left(\mathrm{DBO}_{5}\right)$, demanda química de oxígeno (DQO), tensoactivos aniónicos (analizados como sustancias reactivas al azul de metileno, MBAS por sus siglas en inglés), nitratos $\left(\mathrm{NO}_{3}^{-}\right)$, nitritos $\left(\mathrm{NO}_{2}^{-}\right)$, nitrógeno amoniacal $\left(\mathrm{N}-\mathrm{NH}_{4}{ }^{+}\right)$, fenoles totales, fosfatos $\left(\mathrm{PO}_{4}{ }^{3-}\right)$, hierro (Fe), turbidez, sólidos totales (ST) y material flotante; empleando metodologías validadas y basadas en las normas APHA, (2017). Los parámetros microbiológicos, coliformes totales y fecales (E. coli.), fueron analizados en el Laboratorio de Microbiología de Aguas y Alimentos, DISerLAB de la Pontificia Universidad Católica del Ecuador.

Para evaluar la calidad del agua del río Pita se comparó las concentraciones de los diferentes parámetros físicoquímicos con los límites permisibles establecidos en la normativa ecuatoriana vigente para agua dulce, TULSMA, Libro VI, Anexo 1, Tabla 2.

Finalmente el ICA-NSF de los afluentes del río Pita, fue calculado mediante un promedio aritmético ponderado de las nueve variables, utilizando la ecuación (1):

$$
I C A=\sum_{i=1}^{i=n} Q_{i} w_{i}
$$

Donde: ICA es el índice de calidad del agua, Wi es el factor de la variable i y Qi es el subíndice del parámetro i (Quiroz et al., 2017).

El valor de Qi es estimado a partir de funciones de calidad, ecuaciones o curvas para cada variable. (Quiroz et. al., 2017). Según Castro et. al. (2015), los pesos ICA asignados a cada variable que se utilizan para este índice son: porcentaje de saturación de OD $(0,17)$, coliformes fecales $(0,15), \mathrm{pH}$ $(0,12), \quad \mathrm{DBO}_{5}(0,10), \quad \mathrm{NO}_{3}^{-}(0,10)$, $\mathrm{PO}_{4}^{-3}(0,10)$, temperatura $(0,10)$, turbiedad $(0,08)$ y ST $(0,08)$. De acuerdo a Fernandez \& Solano (2005) y Castro et. al. (2015), la Tabla 2 representa la escala utilizada para interpretar el resultado final de la calidad del agua de acuerdo al ICA-NSF, los resultados son números que cubren el rango de 0 a 100, dónde 0 dictamina la calidad de agua muy pobre y 100 calidad de agua excelente. 


\section{Tabla 2. Calidad del agua asociada al valor del ICA \\ (Escala de clasificación del ICA-NSF)}

\begin{tabular}{lc}
\hline Calidad del Agua & Valor del ICA \\
\hline Excelente & $91-100$ \\
Buena & $71-90$ \\
Media & $51-70$ \\
Mala & $26-50$ \\
Muy Mala & $0-25$ \\
\hline
\end{tabular}

(Fernandez \& Solano, 2005 y Castro et al., 2015)

\section{RESULTADOS}

En el 2018, únicamente se analizó una muestra por punto, mientras que en el 2019 las muestras fueron recolectadas y analizadas por triplicado, en estos casos, se calculó el promedio el cual se reporta en las Tablas 3 y 4 . Se realizaron controles de calidad independientes para cada ensayo (análisis de fortificaciones o estándares puros).
Los resultados obtenidos para las muestras de la sección dentro del Parque Nacional Cotopaxi se presentan en la Tabla 3, y los correspondientes a la sección fuera del Parque Nacional Cotopaxi, se presentan en la Tabla 4. 
EVALUACIÓN DE LA CALIDAD DEL AGUA DEL RÍO PITA (ECUADOR), IMPLICACIÓN PARA LA CONSERVACIÓN DE LA VIDA ACUÁTICA Y SILVESTRE

Tabla 3. Resultados de los parámetros fisicoquímicos de agua del río Pita dentro del Parque Nacional Cotopaxi, aguas arriba y abajo, de cada punto, para comparación con el límite permisible del TULSMA

\begin{tabular}{|c|c|c|c|c|c|c|c|c|c|c|c|}
\hline \multirow{2}{*}{ Parámetro } & \multicolumn{2}{|c|}{ PUNTO 1} & \multicolumn{4}{|c|}{ PUNTO 2} & \multicolumn{4}{|c|}{ PUNTO 3} & \multirow[b]{2}{*}{ LP } \\
\hline & 1B-2018 & $\frac{1 B-2019}{x}$ & $\begin{array}{l}2 A r- \\
2018\end{array}$ & $\underset{\frac{x}{x}}{\operatorname{Ar}-2019}$ & $\begin{array}{c}2 B- \\
2018\end{array}$ & $2 B-\frac{2019}{x}$ & $\begin{array}{l}3 A r- \\
2018\end{array}$ & $\underset{\frac{x}{x}-2019}{2}$ & $\begin{array}{c}3 B= \\
2018\end{array}$ & $\frac{3 B-2019}{x}$ & \\
\hline $\begin{array}{c}\mathrm{Al} \\
\left(\mathrm{mg} \cdot \mathrm{dm}^{-3}\right)\end{array}$ & 0,013 & $<0,010$ & 0,04 & $<0,010$ & 0,037 & $<0,010$ & 0,033 & $<0,010<$ & $<0,010$ & $<0,010$ & 0,1 \\
\hline $\begin{array}{c}\text { As } \\
\left(\mathrm{mg} \cdot \mathrm{dm}^{-3}\right)\end{array}$ & 0,0007 & 0,0005 & 0,0006 & 0,0005 & 0,0008 & 0,0006 & 0,0006 & 0,0010 & 0,0011 & 0,0011 & 0,05 \\
\hline $\begin{array}{c}\mathrm{Ba} \\
\left(\mathrm{mg} \cdot \mathrm{dm}^{-3}\right)\end{array}$ & $<1,0$ & $<1,0$ & $<1,0$ & $<1,0$ & $<1,0$ & $<1,0$ & $<1,0$ & $<1,0$ & $<1,0$ & $<1,0$ & 1 \\
\hline $\begin{array}{c}\mathrm{B} \\
\left(\mathrm{mg} \cdot \mathrm{dm}^{-3}\right)\end{array}$ & $<0,5$ & $<0,5$ & $<0,5$ & $<0,5$ & $<0,5$ & $<0,5$ & $<0,5$ & $<0,5$ & 0,6 & $<0,5$ & 0,75 \\
\hline $\begin{array}{c}\mathrm{CN}^{-} \\
\left(\mathrm{mg} \cdot \mathrm{dm}^{-3}\right)\end{array}$ & $<0,001$ & $<0,001$ & $<0,001$ & $<0,001<$ & $<0,001$ & $<0,001$ & $<0,001$ & $<0,001<$ & $<0,001$ & $<0,001$ & 0,01 \\
\hline $\begin{array}{c}\mathrm{DBO}_{5} \\
\left(\mathrm{mg} \cdot \mathrm{dm}^{-3}\right)\end{array}$ & 6 & $<6$ & 6 & $<6$ & 6 & $<6$ & 7 & $<6$ & $<6$ & $<6$ & 20 \\
\hline $\begin{array}{c}\mathrm{DQO} \\
\left(\mathrm{mg} \cdot \mathrm{dm}^{-3}\right)\end{array}$ & 18 & $<10$ & 11 & 16 & 19 & 12 & $<10$ & $<10$ & $<10$ & 12 & 40 \\
\hline $\begin{array}{l}\text { Fenoles } \\
\left(\mathrm{mg} \cdot \mathrm{dm}^{-3}\right)\end{array}$ & $<0,001$ & $<0,001$ & 0,002 & $<0,001$ & 0,002 & $<0,001$ & $<0,001$ & 0,010 & $<0,001$ & 0,009 & 0,001 \\
\hline $\begin{array}{c}\mathrm{Fe} \\
\left(\mathrm{mg} \cdot \mathrm{dm}^{-3}\right)\end{array}$ & 0,6 & 0,9 & 1,4 & 1,7 & 1,3 & 1,7 & 1,2 & 1,0 & 1,2 & 1,0 & 0,3 \\
\hline $\begin{array}{c}\mathrm{Mn} \\
\left(\mathrm{mg} \cdot \mathrm{dm}^{-3}\right)\end{array}$ & $<0,1$ & $<0,1$ & $<0,1$ & $<0,1$ & $<0,1$ & $<0,1$ & $<0,1$ & $<0,1$ & $<0,1$ & $<0,1$ & 0,1 \\
\hline $\begin{array}{l}\text { Material } \\
\text { Flotante }\end{array}$ & $P$ & $A$ & $P$ & $A$ & $A$ & $A$ & $A$ & $A$ & $A$ & $A$ & $A$ \\
\hline $\begin{array}{c}\mathrm{NO}_{3}^{-} \\
\left(\mathrm{mg} \cdot \mathrm{dm}^{-3}\right)\end{array}$ & $<5,0$ & $<5,0$ & 6,0 & $<5,0$ & 7,2 & $<5,0$ & $<5,0$ & $<5,0$ & $<5,0$ & $<5,0$ & 13 \\
\hline $\begin{array}{c}\mathrm{NO}_{2}^{-} \\
\left(\mathrm{mg} \cdot \mathrm{dm}^{-3}\right)\end{array}$ & $<0,005$ & 0,014 & $<0,005$ & 0,007 & $<0,005$ & 0,014 & 0,006 & $<0,005$ & 0,012 & $<0,005$ & 0,2 \\
\hline $\begin{array}{c}\mathrm{N}-\mathrm{NH}_{4}{ }^{+} \\
\left(\mathrm{mg} \cdot \mathrm{dm}^{-3}\right)\end{array}$ & 0,08 & $<0,05$ & 0,18 & 0,12 & 0,08 & 0,11 & 0,18 & 0,09 & 0,17 & 0,10 & 0,239 \\
\hline $\begin{array}{c}\text { Oxígeno } \\
\text { Disuelto } \\
\% \text { saturación) }\end{array}$ & 108,4 & 107,8 & 112,3 & 103,6 & 111,5 & 97,7 & 111,9 & 106,1 & 112,5 & 109,0 & $>80$ \\
\hline $\begin{array}{l}\mathrm{H} \text { in situ } \\
\text { (Unidad } \\
\text { de } \mathrm{pH} \text { ) }\end{array}$ & 7,7 & 7,0 & 7,5 & 7,1 & 6,7 & 7,5 & 8,3 & 7,6 & 8,4 & 8,3 & $6,5-9$ \\
\hline $\mathrm{T}\left({ }^{\circ} \mathrm{C}\right)$ & 17,4 & 11,2 & 17 & 10,6 & 17,7 & 13,0 & 13,2 & 10,8 & 13,8 & 11,8 & - \\
\hline $\begin{array}{c}\text { Tensoactivos } \\
\text { MBAS } \\
\left(\mathrm{mg} \cdot \mathrm{dm}^{-3}\right)\end{array}$ & $<0,010$ & $<0,010$ & $<0,010$ & 0,012 & 0,012 & 0,011 & 0,012 & $<0,010$ & 0,011 & $<0,010$ & 0,5 \\
\hline
\end{tabular}

Ar: Aguas arriba, B: Aguas Abajo, P: Presencia, A: Ausencia, $\square$ : Promedio, LP: Límite Permisible establecido en el TULSMA, Anexo 1, Tabla 2. 


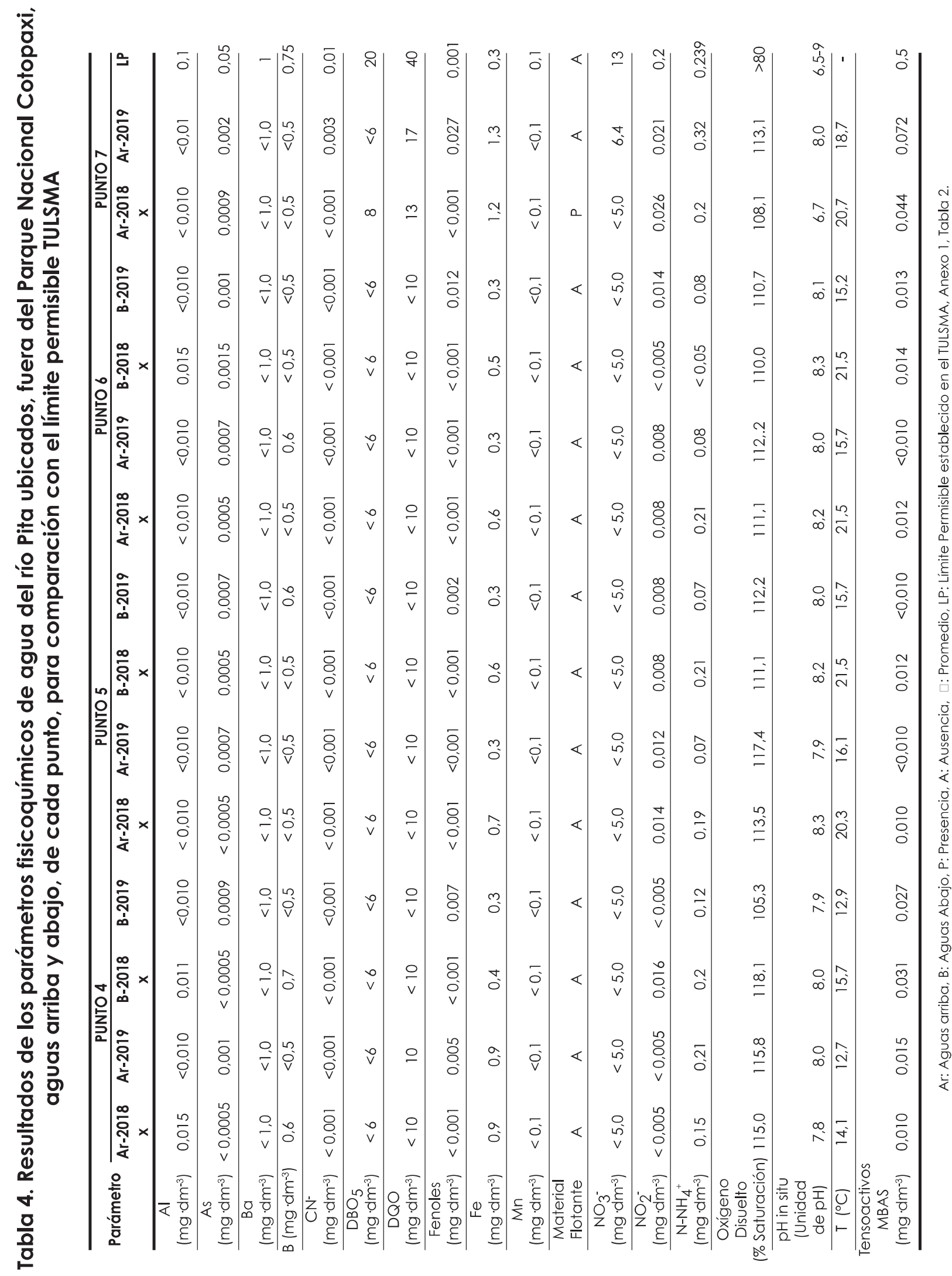


Los resultados del ICA-NSF se presentan en la Tabla 5 correspondientes a las secciones dentro y fuera del Parque Nacional Cotopaxi. La escala utilizada para interpretar el resultado final, se encuentra en el rango de 0 a 100 , dónde " 0 " corresponde a una calidad de agua muy pobre y 100 , corresponde a una calidad de agua excelente.
Tabla 5. Resultados del ICA-NSF de los afluentes del río Pita

\begin{tabular}{|c|c|c|c|c|}
\hline $\begin{array}{l}\text { Río/quebrada } \\
\text { aportante } \\
\text { al río Pita }\end{array}$ & $\begin{array}{l}\text { CA- } \\
\text { INSF } \\
\text { (Ar) }\end{array}$ & $\begin{array}{l}\text { Calidad } \\
\text { del agua }\end{array}$ & $\begin{array}{l}\text { ICA- } \\
\text { ICA- } \\
\text { NSF (B) }\end{array}$ & $\begin{array}{l}\text { Calidad } \\
\text { del agua }\end{array}$ \\
\hline \multicolumn{5}{|c|}{ Sección dentro del Parque Nacional Cotopaxi } \\
\hline $\begin{array}{c}\text { Río } \\
\text { Hualpaloma }\end{array}$ & - & - & 76 & Buena \\
\hline $\begin{array}{l}\text { Quebrada } \\
\text { Madero }\end{array}$ & 72 & Buena & 71 & Buena \\
\hline $\begin{array}{l}\text { Quebrada } \\
\text { La Merced }\end{array}$ & 75 & Buena & 70 & Media \\
\hline \multicolumn{5}{|c|}{ Sección fuera del Parque Nacional Cotopaxi } \\
\hline $\begin{array}{l}\text { Quebrada } \\
\text { Chorro }\end{array}$ & 70 & Media & 70 & Media \\
\hline $\begin{array}{l}\text { Quebrada } \\
\text { Cariacu }\end{array}$ & 73 & Buena & 70 & Media \\
\hline $\begin{array}{l}\text { Río Guapal } \\
\text { Confluencia } \\
\text { con el río }\end{array}$ & 70 & Media & 66 & Media \\
\hline San Pedro & 60 & Media & - & - \\
\hline
\end{tabular}

Ar: Aguas arriba, B: Aguas Abajo, ICA-NSF: Índice de calidad del agua de la Fundación Nacional de Saneamiento

\section{DISCUSIÓN}

Los resultados de la presente investigación (Tabla 3), presentan concentraciones de: $\mathrm{Al}, \mathrm{As}, \mathrm{Ba}, \mathrm{B}, \mathrm{Mn}, \mathrm{CN}$ libre, $\mathrm{DBO}_{5}, \mathrm{DQO}$, tensoactivos aniónicos, $\mathrm{NO}_{3}{ }^{-}, \mathrm{NO}_{2}{ }^{-}, \mathrm{N}-\mathrm{NH}_{4}{ }^{+}$, oxígeno disuelto y $\mathrm{pH}$, inferiores a los límites permisibles correspondientes establecidos en la normativa ecuatoriana (TULSMA ,2017)

\section{Análisis fisicoquímicos de los afluen- tes del río Pita ubicados dentro del parque Nacional Cotopaxi}

La concentración más alta encontrada para el Al en el 2018, correspondió a la quebrada Madero, aguas arriba (Punto 2Ar), con un valor de $0,040 \mathrm{mg} \cdot \mathrm{dm}^{-3}$. En el 2019 todos los resultados estuvieron por debajo del límite de cuantificación del método utilizado, la presencia de este metal se atribuye a la meteorización de rocas silicatadas presentes en el río 
(Alarcón, Leal, Martín, Miranda, \& Benavides, 2014). En cuanto a las concentraciones de As, los valores más altos encontrados tanto en el 2018 como en el $2019(0,0011$ $\left.\mathrm{mg} \cdot \mathrm{dm}^{-3}\right)$, corresponden la quebrada la Merced, aguas abajo (Punto 3B). En ambos metales, los valores se encuentran por debajo de los límites máximos permisibles de: $0,1 \mathrm{mg} \cdot \mathrm{dm}^{-3}$ para Al y $0,05 \mathrm{mg} \cdot \mathrm{dm}^{-3}$ para As. Estos elementos son indicadores ambientales de contaminación antrópica debido a su proveniencia de descargas de aguas residuales industriales y de producción animal (Cao et al., 2018; Nandana y col., 2012).

Para el caso del B en el 2018, se obtuvo como valor máximo $0,6 \mathrm{mg} \cdot \mathrm{dm}^{-}$ ${ }^{3}$ en el punto de la quebrada La Merced, aguas abajo (Punto 3B), sin embargo, se encuentra por debajo del límite máximo permitido $(0,75$ $\left.\mathrm{mg} \cdot \mathrm{dm}^{-3}\right)$. Para las muestras recolectadas en el 2019 las concentraciones estuvieron por debajo de $0,5 \mathrm{mg} \cdot \mathrm{dm}^{-}$ ${ }^{3}$ en todos los puntos. La presencia de este metal, se atribuye a la descomposición de minerales como los granitos y boratos.

El Fe supera el límite máximo permisible de $0,3 \mathrm{mg} \cdot \mathrm{dm}^{-3}$ en todos los puntos, encontrándose concentraciones entre 0,6 y $1,7 \mathrm{mg} \cdot \mathrm{dm}^{-3}$ tanto en el 2018 y 2019, la presencia de este metal es de origen natural, proveniente de la disolución de las rocas y minerales, por lo tanto su incremento se atribuye a eventos de lluvia que causen mayor escurrimiento superficial o arrastre de sedimento (MAE, 2012). Para el caso de los tensoactivos aniónicos (MBAS), los resultados presentaron valores inferiores a 0,012 $\mathrm{mg} \cdot \mathrm{dm}^{-3}$, siendo los valores más altos en la quebrada Madero, aguas abajo (Punto 2B) y La Merced, aguas arriba (Punto 3Ar) durante el 2018; mientras que en el 2019, en la quebrada Madero aguas arriba y aguas abajo (Punto $2 \mathrm{Ar}$ y $2 \mathrm{~B}$ ) presentaron valores de 0,012 y $0,011 \mathrm{mg} \cdot \mathrm{dm}^{-3}$; no obstante, estos valores se encuentran por debajo del límite máximo permisible, $0,5 \mathrm{mg} \cdot \mathrm{dm}^{-3}$. Este contaminante es de origen sintético (Cabanes, 2014), por lo cual su presencia puede atribuirse a la descargas de residuos domésticos de haciendas aledañas, en la cual se utilicen detergentes de uso doméstico (F. Ríos, 2012).

El nitrógeno fue cuantificado en forma de nitrógeno amoniacal, nitritos y nitratos. La concentración más alta de $\mathrm{NO}_{3}{ }^{-}$en el 2018 fue de 7,2 
$\mathrm{mg} \cdot \mathrm{dm}^{-3}$, encontrándose el valor más alto en la quebrada Madero aguas abajo (Punto 2B), mientras que el 2019 todos los valores estuvieron debajo del límite de cuantificación. Para el caso de las concentraciones de $\mathrm{NO}_{2}^{-}$, fueron inferiores a 0,012 $\mathrm{mg} \cdot \mathrm{dm}^{-3}$ en el 2018, siendo el de mayor concentración el punto 3B, quebrada La Merced aguas abajo. En el 2019 las concentraciones más altas se presentaron en el río Hualpaloma (Punto 1B) y quebrada Madero, aguas abajo (Punto 2B) con valores de $0,014 \mathrm{mg} \cdot \mathrm{dm}^{-3}$. El nitrógeno amoniacal, en el 2018, presentó valores entre 0,08 y 0,18 $\mathrm{mg} \cdot \mathrm{dm}^{-3}$; mientras que en el $2019 \mathrm{el}$ valor máximo fue de $0,012 \mathrm{mg} \cdot \mathrm{dm}^{-3}$ en la quebrada Madero, aguas arriba (Punto 2Ar). Estos tres parámetros se encuentran por debajo de los límites máximos permisibles correspondientes: $13 \mathrm{mg} \cdot \mathrm{dm}^{-3}$ de $\mathrm{NO}_{3}{ }^{-}, 0,2 \mathrm{mg} \cdot \mathrm{dm}^{-}$ ${ }^{3}$ de $\mathrm{NO}_{2}^{-}$y $0,239 \mathrm{mg} \cdot \mathrm{dm}^{-3}$ de nitrógeno amoniacal. La presencia de este último en aguas superficiales se debe a la degradación natural de la materia orgánica (acumulación de excretas de animales), siendo una etapa del ciclo del nitrógeno, componente transitorio del agua influenciado por la actividad biológica. También puede deberse a activida- des antropogénicas como: descargas de aguas residuales, domésticas e industriales, uso excesivo de fertilizantes en la agricultura (Barrenechea, n.d.). En ambos casos, el nitrógeno amoniacal se origina de la degradación del nitrógeno orgánico y, este a su vez, por acción bacteriana, se oxida gradualmente a nitritos y finalmente a nitratos (González, 2015).

Generalmente, luego de los procesos de autodepuración del agua, el pH tiende a ser ácido, se presume que estos procesos son mínimos ya que en las muestras analizadas tuvieron valores de $\mathrm{pH}$ entre 6,7 y 8,3 en el 2018, y en el 2019 los valores estuvieron entre 7,0 y 8,3 , rango de $\mathrm{pH}$ neutro a levemente alcalino para los dos casos.

Por otra parte, los parámetros indicadores de calidad de agua como la $\mathrm{DBO}_{5}$ y DQO presentan resultados menores a 7 y $19 \mathrm{mg} \cdot \mathrm{dm}^{-3}$ respectivamente en el 2018, y menores a 6 y $12 \mathrm{mg} \cdot \mathrm{dm}^{-3}$ en el 2019, respectivamente. Encontrándose por debajo de los límites máximos permisibles correspondientes de $20 \mathrm{mg} \cdot \mathrm{dm}^{-3}$ para la $\mathrm{DBO}_{5}$ y de $40 \mathrm{mg} \cdot \mathrm{dm}^{-3}$ para la DQO. Estos parámetros se relacio- 
nan con el contenido de materia orgánica y compuestos que puedan ser degradados biológicamente $\left(\mathrm{DBO}_{5}\right.$, actividad microbiana), o químicamente (DQO, oxido reducción u oxidación) (Rivera, Encina, \& Muñoz, 2004).

El porcentaje de saturación de OD es un indicador de procesos de aireación y fotosíntesis de algas. La baja concentración de OD limita la capacidad de purificación de las aguas naturales (Rivera, Encina, Muñoz-Pedreros, et al., 2004; Spiegel \& Maystre, 2007). De acuerdo a los resultados expuestos en la Tabla 3, las muestras presentan una saturación óptima de oxígeno disuelto encontrándose sobre el $80 \%$ en todos los casos, presentándose en el 2018 y en el 2019 el mayor porcentaje de saturación de $\mathrm{OD}$ en el punto $3 \mathrm{~B}$ de la quebrada La Merced, aguas abajo, con $112,5 \%$ y $109,0 \%$ respectivamente.

Respecto al parámetro material flotante de origen antrópico, en los criterios de calidad para agua dulce se establece como "ausencia", entendiéndose como la inexistencia de materiales visibles no naturales. En el
2018, en los puntos 1 y 2, río Hualpaloma y quebrada Madero, aguas arriba, se determinaron partículas negras, las cuales corresponden a fragmentos de hojas o material orgánico debido a la exposición al ser un canal abierto. En el 2019 las muestras no presentaron material flotante, cumpliendo la normativa vigente.

Otros compuestos considerados contaminantes como los fenoles, se encontraron en las muestras correspondientes al punto 2, quebrada El Madero tanto aguas arriba como aguas abajo, con concentraciones de 0,002 $\mathrm{mg} \cdot \mathrm{dm}^{-3}$ en el 2018; mientras que en el 2019 las concentraciones más altas fueron encontradas en el punto 3, quebrada La Merced, aguas arriba y abajo con valores de 0,010 y 0,009 $\mathrm{mg} \cdot \mathrm{dm}^{-3}$, respectivamente, superando el límite máximo permisible $(0,001$ $\left.\mathrm{mg} \cdot \mathrm{dm}^{-3}\right)$. Esta relativa contaminación puede atribuirse a procesos naturales, como la formación de fenol y $p$-cresol durante la descomposición de materia orgánica o la síntesis de fenoles clorados por los hongos y las plantas (Michałowicz \& Duda, 2007; Alcaraz, Fabiano, \& Cámara, 2012). 
Respecto a la temperatura, este parámetro en aguas superficiales fluctúa de acuerdo con las estaciones del año, en invierno con $5,0^{\circ} \mathrm{C}$ y en primavera-verano con $21,0^{\circ} \mathrm{C}$ (Rivera, Encina, Muñoz-Pedreros, et al., 2004). Desde el punto de vista de la vida acuática, no debe aumentar el valor natural en más de $3,0^{\circ} \mathrm{C}$, y con respecto al contacto directo se puede tolerar hasta un máximo de $30^{\circ} \mathrm{C}(\mathrm{Ri}-$ vera, Encina, Muñoz-Pedreros, et al., 2004). De acuerdo a los resultados de la Tabla 3, las temperaturas estuvieron entre $13,2^{\circ} \mathrm{C}$ y $17,4{ }^{\circ} \mathrm{C}$ en el año 2018 , y entre $10,6{ }^{\circ} \mathrm{C}$ a $13,0{ }^{\circ} \mathrm{C}$ en el año 2019. En base a lo anterior, las muestras se encuentran dentro de los requisitos mínimos para la vida acuática y silvestre en aguas dulces establecidos en la norma ecuatoriana.

\section{Análisis fisicoquímicos de los afluen- tes del río Pita ubicados fuera del parque Nacional Cotopaxi}

De acuerdo a la Tabla 4, las concentraciones de $\mathrm{Al}$ fueron menores a $0,015 \mathrm{mg} \cdot \mathrm{dm}^{-3}$ en el $2018 \mathrm{y}<0,010$ $\mathrm{mg} \cdot \mathrm{dm}^{-3}$ en el 2019 , en comparación con la concentración de los afluentes ubicados dentro del parque $(0,040$ $\mathrm{mg} \cdot \mathrm{dm}^{-3}$ ) debe considerarse, que el caudal del río es menor con respecto al de fuera del parque.

Con respecto al As, los puntos ubicados fuera del Parque Nacional Cotopaxi presentan concentraciones menores a $0,0015 \mathrm{mg} \cdot \mathrm{dm}^{-3}$ en el 2018 y en el 2019 menores a $0,0010 \mathrm{mg} \cdot \mathrm{dm}^{-3}$. Para este caso cabe mencionar que en los puntos 4 y 5 (Tabla 4), aguas abajo de la quebrada Chorro y aguas arriba de la quebrada Cariacu, se encuentra la planta de tratamiento de agua potable, EPMAPS, la misma que realiza captación de agua y un pretratamiento de potabilización. A partir del punto 6 correspondiente a un área recreativa, "cascadas Rumibosque", la concentración de As se incrementa levemente. Sin embargo las concentraciones de As de las muestras tanto dentro como fuera del Parque Nacional Cotopaxi se encuen tran por debajo de los límites establecidos en el TULSMA.

Con respecto a los resultados obtenidos de boro en el punto 4, quebrada Chorro, aguas abajo, ubicado fuera del Parque Nacional Cotopaxi, presenta la mayor concentración siendo $0,7 \mathrm{mg} \cdot \mathrm{dm}^{-3}$ en el 2018 , mientras que 
en el 2019 el punto 5 y 6, quebrada Cariacu, aguas abajo y río Guapal, aguas arriba presentan $0,6 \mathrm{mg} \cdot \mathrm{dm}^{-3}$. Este metal se encuentra presente principalmente en la cercanía de zonas geotérmicas (Mancilla-Villa, Bautista-Olivas, \& Ramírez-Ayala, 2014), como es el volcán Cotopaxi, así mismo, con respecto al punto 3, Quebrada La Merced, ubicado dentro de parque, presenta $0,6 \mathrm{mg} \cdot \mathrm{dm}^{-3}$ de $B$, sin embargo estos valores se encuentran por debajo del LP establecidos en la norma.

Las concentraciones de Fe de las muestras ubicadas fuera del parque presentaron valores entre 0,4 y 1,2 $\mathrm{mg} \cdot \mathrm{dm}^{-3}$ en el 2018, y entre 0,3 y 1,3 $\mathrm{mg} \cdot \mathrm{dm}^{-3}$ en el 2019 , presentándose la concentración más alta en el punto 7, antes de la confluencia con el río San Pedro. Este incremento está relacionado a la influencia de actividades antropogénicas, a diferencia del contenido de este metal dentro del Parque Nacional, el cual está relacionado a fuentes naturales.

Para el caso de los tensoactivos aniónicos (MBAS), en el 2018, los valores más altos se encontraron en los puntos $4 \mathrm{~B}$ y $7 \mathrm{Ar}$, quebrada Chorro, aguas abajo y confluencia con el río San Pedro, los resultados fueron 0,031 y $0,044 \mathrm{mg} \cdot \mathrm{dm}^{-3}$ respectivamente. En el 2019, los valores fueron 0,027 y $0,072 \mathrm{mg} \cdot \mathrm{dm}^{-3}$ en los mismos puntos. Se presume que las altas concentraciones se deben por encontrarse en áreas turísticas y urbanas. Para todos los casos, los valores se encuentran por debajo del límite permisible de $0,5 \mathrm{mg} \cdot \mathrm{dm}^{-3}$. Este contaminante pudo estar presente debido a la descarga de residuos domésticos (F. Ríos, 2012).

Con relación a la concentración de cianuro libre los resultados de las muestras recolectadas en el 2018 y 2019 dentro del Parque Nacional Cotopaxi se encontraron debajo de límite máximo permisible, 0,01 $\mathrm{mg} \cdot \mathrm{dm}^{-3}$, al igual que las muestras recolectadas en el 2018 fuera del parque. Sin embargo para el 2019 se tuvo un valor de $0,003 \mathrm{mg} \cdot \mathrm{dm}^{-3} \mathrm{de}$ cianuro libre en el punto 7 , antes de la confluencia del río San Pedro, se presume que este contaminante proviene de descargas industriales (Martinez, Ferro, \& Pablos, 2016).

Las concentraciones de $\mathrm{NO}_{3}^{-}$en el 2018 se encuentran por debajo de 5 
$\mathrm{mg} \cdot \mathrm{dm}^{-3}$, a comparación de las muestras ubicadas dentro del Parque Nacional Cotopaxi, en el 2019 se tuvo un valor de $6,4 \mathrm{mg} \cdot \mathrm{dm}^{-3}$ en el punto 7 . En ningún caso se supera el límite permisible establecido, 13 $\mathrm{mg} \cdot \mathrm{dm}^{-3}$.

Las concentraciones de $\mathrm{NO}_{2}{ }^{-}$, fueron menores a $0,026 \mathrm{mg} \cdot \mathrm{dm}^{-3}$, siendo el de mayor concentración el punto 7Ar, en el 2018 y 2019. Comparando con las muestras recolectadas dentro del parque, las muestras fuera del parque presentan mayor concentración de $\mathrm{NO}_{2}{ }^{-}$, sin embargo se encuentran por debajo del límite permisible establecido en la normativa ecuatoriana, $0,2 \mathrm{mg} \cdot \mathrm{dm}^{-3}$.

Las concentraciones de nitrógeno amoniacal son inferiores a 0,21 $\mathrm{mg} \cdot \mathrm{dm}^{-3}$ en el 2018 , siendo los de mayores concentraciones los puntos 5 y 6 , pertenecientes a aguas abajo de la quebrada Chorro y aguas arriba del río Guapal. Para el 2019, el punto 7 Ar presentó la concentración más alta, 0,32 $\mathrm{mg} \cdot \mathrm{dm}^{-3}$. En general, las muestras fuera del parque presentan mayores concentraciones en comparación con las muestras dentro del parque, este parámetro se en- cuentra directamente relacionado con la cercanía con zonas urbanas y confluencia de actividades antropogénicas. Sin embargo, este parámetro analizado se encuentra por debajo del límite máximo permisible 0,239 $\mathrm{mg} \cdot \mathrm{dm}^{-3}$.

$\mathrm{El} \mathrm{pH}$ de las muestras fuera del parque en el 2018 presentó valores entre 6,7 y 8,3 y en el 2019 valores entre 7,9 a 8,1, similares a las muestras tomadas dentro del parque. Para todos los casos se observa el cumplimiento con los límites permisible expuestos en la normativa, de 6,5 a 9,0 unidades de $\mathrm{pH}$.

Por otra parte, la $\mathrm{DBO}_{5}$ y DQO en el 2018, presentaron resultados menores a 8 y a $13 \mathrm{mg} \cdot \mathrm{dm}^{-3}$ respectivamente, y en el 2019 el DQO fue 17 $\mathrm{mg} \cdot \mathrm{dm}^{-3}$, presentándose los valores más altos en el punto 7; sin embargo se encuentran por debajo de los límites máximos permisibles de 20 $\mathrm{mg} \cdot \mathrm{dm}^{-3}$ y de $40 \mathrm{mg} \cdot \mathrm{dm}^{-3}$ respectivamente.

Las muestras recolectadas dentro del parque presentan mayor concentración debido al menor caudal del río Pita lo que, ocasiona mayor presen- 
cia de materia orgánica y compuestos que puedan ser degradados biológica o químicamente.

De acuerdo a los resultados expuestos en la Tabla 4, las muestras presentan una saturación de OD óptima encontrándose sobre el 80 \%. En el año 2018 el valor más alto fue $118,1 \%$ perteneciente al punto $4 \mathrm{~B}$, quebrada Chorro, aguas abajo, mientras que en el 2019 fue de 117,4 \% en el punto $5 \mathrm{Ar}$, quebrada Cariacu, aguas arriba. Al comparar las concentraciones de saturación de OD, las muestras tomadas dentro del parque presentan menor porcentaje de saturación, este incremento puede deberse a las diferentes caídas de agua existentes.

En el 2018 y 2019 las muestras recolectadas dentro y fuera del Parque Nacional, no presentaron material flotante de origen antrópico, a excepción de la muestra recolectada en el punto 7, confluencia del río San Pedro, en el 2018. En esta se identificó la presencia de partículas visibles no naturales de color blanco y negro.

Para el caso del parámetro fenoles, en el 2018, las muestras recolectadas fuera del parque nacional Cotopaxi no presentaron concentraciones de este contaminante. Para el 2019, las muestras de los puntos 4, 5, 6 y 7 presentan concentraciones de fenoles, siendo la mayor el punto 7 , con $0,027 \mathrm{mg} \cdot \mathrm{dm}^{-3}$. La presencia de este contaminante dentro del parque es de origen natural, mientras que la presencia en la muestras fuera del parque se atribuye a contaminación industrial.

De acuerdo a los resultados de la Tabla 4, en el año 2018, la temperatura presentó valores en el rango de $14,1^{\circ} \mathrm{C}$ y $21,5^{\circ} \mathrm{C}$, mientras que en el 2019 la temperatura estuvo entre $12,7^{\circ} \mathrm{C}$ y $18,7^{\circ} \mathrm{C}$. Con respecto a las aguas del río Pita ubicadas dentro del Parque Nacional Cotopaxi, estas presentan temperaturas más bajas, debido a la altitud y la cercanía del volcán Cotopaxi, sin embargo, no supera el valor tolerable de $30^{\circ} \mathrm{C}$.

\section{Índice de Calidad del Agua del río Pita}

De acuerdo a la Tabla 5, los ICA-NSF del río Hualpaloma, la quebrada Madero y quebrada la Merced aguas arriba, presentan índices de calidad 
"buena", sin embargo para el caso del punto aguas abajo de la quebrada La merced, presenta un índice de calidad " medio".

El ICA-NSF de la quebrada Chorro, reporta una calidad " media" al igual que para la quebrada Cariacu, aguas abajo y río Guapal tanto aguas arriba como aguas abajo.
El punto 7 perteneciente a la confluencia del río San Pedro presenta el menor ICA-NSF (60), que a pesar que lo califica como agua de calidad media, es evidente que es el más afectado por la presencia de contaminantes.

\section{CONCLUSIONES}

Los resultados obtenidos de los parámetros: Al, As, Ba, cianuro libre, DQO, $\mathrm{DBO}_{5}$, tensoactivos MBAS, $\mathrm{Mn}, \mathrm{N}-\mathrm{NH}_{3}, \mathrm{~B}, \mathrm{NO}_{3}, \mathrm{NO}_{2}$, porcentaje de saturación de OD, y sólidos suspendidos totales, de este estudio, presentan concentraciones por debajo del límite establecido para agua dulce según la Tabla 2 del Anexo 1 del TULSMA, a excepción de los parámetros: fenoles y $\mathrm{Fe}$, los cuales sobrepasan los límites máximos establecidos.

Debería considerarse la revisión del límite máximo permisible del $\mathrm{Fe}$, ya que este metal se origina por la ero- sión del suelo, y puede llegar a los acuíferos aledaños.

El índice de calidad del agua aplicado, permite evidenciar que las muestras recolectadas dentro del parque Nacional Cotopaxi no han sido mayormente influenciadas por actividades antropogénicas y presentan una calidad de agua buena, contrariamente a lo que se evidencia en las muestras recolectadas fuera del parque, las cuales, con una calidad de agua media, indican la influencia de actividades antropogénicas de las zonas urbanas e industriales aledañas. 


\section{LISTA DE REFERENCIAS}

Alarcón, T., Leal, L., Martín, I., Miranda, S., \& Benavides, A. (2014). Arsénico en agua. Cimav, (2).

Alcaraz, M. R., Fabiano, S. N., \& Cámara, M. S. (2012). Determinación De Contenido Fenólico Total En Agua Superficial De Distintos Puntos De La Provincia De Santa Fe - Argentina - Haciendo Uso De Un Biosensor Enzimático Mediante Calibración Multivariada Por Cuadrados Parciales Mínimos, Pls. Septimo Congreso de Medio Ambiente.

Barrenechea, A. M. (n.d.). Capítulo 1 Aspectos Fisicoquímicos De La Calidad Del Agua.

Blanco, J. F. (2009). Características Físico-Químicas De Las Quebradas Del Parque Nacional Natural Gorgona, Pacífico Colombiano. Actual Biol, 31(91), 123-140.

Cabanes, A. (2014). Surfactants in textiles. Tecnlogía, 3, 137-151.

Campaña, A., Gualoto, E., \& Chiluisa-Utreras, V. (2017). Evaluación físico-química y microbiológica de la calidad del agua de los ríos Machángara y Monjas de la red hídrica del distrito metropolitano de Quito. Bionatura, 2(2), 305-310. https://doi.org/10.21931/RB/2017.02.02.6

Cao, Y., Lei, K., Zhang, X., Xu, L., Lin, C., \& Yang, Y. (2018). Contamination and ecological risks of toxic metals in the Hai River, China. Ecotoxicology and Environmental Safety, 164, 210-218. https://doi.org/10.1016/j.ecoenv.2018.08.009

Castro, M., Almeida, J., Ferrer, J., \& Diaz, D. (2015). Indicadores de la calidad del agua: evolución y tendencias a nivel global. Ingeniería Solidaria, 9(17). https://doi. org/10.16925/in.v9i17.811

Código Orgánico del Ambiente. (2017). Codigo Organico del Ambiente, 92. Retrieved from http://www.competencias.gob.ec/wp-content/uploads/2017/06/05NOR2017COA.pdf 
EVALUACIÓN DE LA CALIDAD DEL AGUA DEL RÍO PITA (ECUADOR), IMPLICACIÓN PARA LA CONSERVACIÓN DE LA VIDA ACUÁTICA Y SILVESTRE

Simbaña et. al. 71-93

Empresa Pública Metropolitana de Agua Potable y Saneamiento. (2016). Manejo y Conservación de las cuencas aportantes a los sistemas de agua potable de la EPMAPS.

Fernandez, J., \& Solano, F. (2005). Indices de Calidad y de Contaminación del Agua. Índices de Calidad (ICAs) y de Contaminación (ICOs) Del Agua de Importancia Mundial.

Fondo para la Protección del Agua (FONAG). (2014). Caracterización biofísica y socioeconómica de la cuenca alta del río Guayllabamba, con énfasis en las subcuencas de los ríos Pita y San Pedro y las microcuencas de los ríos orientales Papallacta y Antisana, 92.

González, L. (201). Nitrógeno amoniacal, importancia de su determinación, Mente \& Materia, 4(1), 12-13.

Hernandez, D., \& Lara, D. (2005). Facultad De Ingeniería Geográfica Y Del Los Rios Pita Y San Pedro, 177. Retrieved from www.espe.edu.ec

INEN. (2013). Norma Técnica Ecuatoriana NTE INEN 2169: 2013 AGUA. Calidad del Agua. Muestreo, Manejo y Conservación de Muestras. Norma Técnica Ecuatoriana (Vol. First Edit). Ecuador.

Jacobsen, D. (2003). Los ríos de la Sierra ecuatoriana son más sensibles a la contaminación. Desafío. Retrieved from http://repositorio.educacionsuperior.gob.ec/handle $/ 28000 / 614$

Leandro, H., Coto, J., \& Salgado, V. (2010). Microcuenca Iv Del Río Virilla. Uniciencia, $24,69-74$.

Liñero, I., Balarezzo, V., Eraso, H., Pacheco, F., Ramos, C., Muzo, R., \& Calva, C. (2016). Calidad del agua de un río andino ecuatoriano a través del uso de macroinvertebrados. Cuadernos de Investigación UNED, 8(1), 69-75.

Mancilla-Villa, O. R., Bautista-Olivas, A. L., \& Ramírez-Ayala,. C. (2014). Contenido de boro en el agua superficial de Puebla, Tlaxcala y Veracruz, Tecnologia y ciencias del agua, 5(5), 97-109. 
Maritza, M., Aguirre, R., Eddi, S., Vanegas, A., li, C., García, C. N., \& li, Á. (2016). Aplicación del Índice de Calidad del Agua ( ICA ). Caso de estudio: Lago de Izabal, Guatemala, Revista ciencias técnicas agropecuarias, 25(2), 39-43.

Martinez, M., Ferro, E., \& Pablos, F. De. (2016). Evaluation of Free Cyanide in Superficial Waters of River Paraguay Nearby a Steel Industry, 88-94. https://doi.org/10. 1111/j.1467-9388.2005.00419.x

Menéndez, A. N. (2010). Transporte de contaminantes en el Medio Acuático. Universidad Tenológica Nacional, Maestría En Ingeniería Ambiental, 123.

Michałowicz, J., Duda, W., \& Michalowicz, J. (2007). Phenols-sources and toxicity. Pol. J. Environ. Stud, 16(3), 347-362. Retrieved from http://www.pjoes.com/pdf/16.3/ 347-362.pdf

Ministerio del Ambiente. (2012). Plan de Manejo Adaptativo del sistema de agua potable Pita- Puengasí y sus cuencas abastecedoras, 40-70. Retrieved from http://www.ambiente.gob.ec/wp-content/uploads/downloads/2014/07/Plan-de-Manejo-Adaptativo-SAP-Pita.pdf

Ministerio del Ambiente. (2017). Texto Unificado de Legislación Secundaria del Ministerio del Ambiente, 14(1), 25-33. https://doi.org/10.1017/CBO9781107415324 .004

Paul, D. (2017). Research on heavy metal pollution of river Ganga: A review. Annals of Agrarian Science, 15(2), 278-286. https://doi.org/10.1016/j.aasci.2017.04.001

Quiroz, L., Izquierdo, E., \& Menendez, C. (2017). Aplicación del índice de calidad de agua en el río Portoviejo, Ecuador, XXXVIII(3), 41-51.

Ríos, F. (2012). Comportamiento ambiental de Tensoactivos comerciales: Biodegradabilidad, Toxicidad y ozonización. Retrieved from http://depa.fquim.unam.mx/fina/ presenta/tensoactivos.pdf

Ríos, L., Anaya, L., López-ríos, O., \& Lechuga-anaya, M. C. M. (2001). Contaminantes en los cuerpos de agua del sur de Sonora, Salud pública de México, 43(4). 
Rivera, R., Encina, F., Muñoz-Pedreros, A., \& Mejias, P. (2004). La Calidad de las Aguas en los Ríos Cautín e Imperial, IX Región-Chile. Información Tecnológica, 15(5), 89-101. https://doi.org/10.4067/S0718-07642004000500013

Rivera, R., Encina, F., \& Muñoz, A. (2004). La Calidad de las Aguas en los Resesrva Nacional Malleco en la IX Regió de Chile. Informacion Tecnologica, 15(5), 89-102. https://doi.org/10.4067/S0718-07642004000500013

Spiegel, J., \& Maystre, L. (2007). Control y Prevención de la Contaminación Ambiental. In El Medio Ambiente (25), 508-512. 